\section{BACKSTEP: A simple program for backward-selection multiple regression}

\section{BERNARD S. GORMAN and LOUIS H. PRIMAVERA Hofstra University, Hempstead, New York 11550}

Although there are many programs and algorithms for multiple regression, only a few exist for stepwise multiple regression. The present BACKSTEP program uses a backward variable-selection algorithm that starts with a set of $n$ variables and, on the basis of a statistical usefulness criterion, selectively deletes one variable at a time to form progressively smaller subsets of predictors. Although backward selection is not as frequently used as forward selection, the method leads to fairly simple programs. The present program is no exception to this notion, as it can generate a large number of useful statistics using fewer than 200 FORTRAN statements and can solve a 20 -variable problem using less than 24 kbytes of storage. Central to the procedure is the efficient use of the inverse of the matrix of correlations among both the criterion and predictor variables.

Algorithm. Although several articles have discussed the derivation of regression statistics from the inverse of a matrix of correlations among the predictor and criterion variables (e.g., Dubois \& Manning, 1960; Fox, 1968), most textbooks and articles on multiple regression generally employ $R^{-1}$, the inverse of the correlation matrix among the predictor variables. A column vector of beta weights, $b$, is then computed by the formula $b=R^{-1} v$, where $v$ is a column vector of zero-order correlations of each of the predictors with the criterion variable. The squared multiple-correlation coefficient, $R^{2}$, is then found by the formula $R^{2}=b^{\prime} v=$ $\mathrm{v}^{\prime} \mathrm{b}$. The BACKSTEP algorithm, however, employs the matrix $Q$, which is the inverse of the matrix of correlations among both the predictors and the criterion.

Through the use of the inverse matrix, $Q$, all of the important multiple-regression statistics can be derived for ordinary multiple linear regression, as well as for stepwise regression. Let the subscript $p$ stand for any predictor variable, let the subscript $c$ stand for the criterion variable, and let $q_{i j}$ stand for the element at Row $\mathrm{i}$ and Column $\mathrm{j}$ of $\mathrm{Q}$.

Using this notation, the following statistics may be computed. (1) The squared multiple correlation, $R^{2}$, is obtained by $R^{2}=1-\left(1 / q_{c c}\right)$, where $q_{c c}$ is the diagonal element in Column $c$ of $Q$. (2) The beta weight for any predictor, $\beta$, is $\beta_{p}=-q_{p c} /\left(1 / q_{c c}\right)$. The partial correlation between Variable $\mathrm{p}$ and the criterion $\mathrm{c}$ is $p t_{p c}=-q_{p c} / \sqrt{q_{p p} q_{c c}}$. (4) The squared semipartial correlation between any predictor and the criterion is $u_{p c}=\left[p t_{p c} /\left(1-p^{2}{ }_{p c}\right)\right] / q_{c c}$. The symbol $u_{p c}$ has been used because the squared semipartial correlation (part correlation) has been called the "usefulness" or unique variance of Variable $p$ (Darlington, 1968). It represents the actual proportion by which $R^{2}$ would decrease if $p$ was deleted from the subset of predictors at the present step of the regression analysis. (5) The significance test for the null hypothesis that the beta weight for Variable $p$ does not differ significantly from a zero-regression weight can be found by $F_{p}=u_{p c} q_{c c}(m-n-1)$, where $m$ is the number of subjects and $n$ is the number of predictors used at any given step.

The statistics derived thus far could be used in both ordinary and stepwise regression. In order to perform backward-selection regression, BACKSTEP searches for the variable at each step that has the smallest squared semipartial correlation with the criterion, eliminates it from the predictor set, and then adjusts the elements of the inverse matrix by the use of a "sweep operator" (Overall \& Klett, 1972). Let d represent a variable to be deleted and let $i$ and $j$ be any two variables in $Q$. Then the revised inverse element $q_{i j}^{*}$ will be $q_{i j}^{*}=q_{i j}-\left(q_{i d} q_{j d} / q_{d d}\right)$. By using the sweep operation formulation above, the BACKSTEP program avoids the need to compute a new inverse at each step. The regression statistics are then obtained as before from the revised inverse, and the procedure is repeated for each step until either all of the predictors have been eliminated or the maximum number of steps requested by the user has been reached.

Input. BACKSTEP will permit multiple analyses in a single run. Users have the option of either entering raw data for each subject or entering a previously computed correlation matrix. Each data set requires a parameter card that sets the number of subjects, the number of variables, the desired number of steps, and other options. In addition, a variable format card is required for each data deck. A blank card placed after the last data deck will terminate the run.

Output. The program outputs the following information: (1) the means and standard deviations of each variable, if raw data were supplied; (2) the correlation matrix, its inverse, and its determinant; (3) F tests for testing the significance of differences between the obtained $R^{2}$ and an $R^{2}$ of zero, $F$ tests for the differences in $R^{2}$ from step to step, and $F$ tests for the significance of each beta weight; (4) beta weights, partial correlations, squared semipartial correlations, and rawscore regression weights for each variable at each step; (5) the raw-score regression constant or intercept term, if raw scores were entered.

Computer and Language. BACKSTEP is written in a very portable version of FORTRAN IV and has run successfully on an IBM 370/138 using 24 kbytes for a problem using 19 predictors. The program may be easily expanded by changing a few DIMENSION statements. 
The only modification that might be needed for nonIBM systems involves the conversion of some labels that appear in some format statements from apostrophe notation to $\mathrm{H}$ notation.

Program Limitations. At present, the parameter card allows up to 9,999 subjects and 20 variables. However, the program is essentially unlimited with respect to the number of subjects and can be easily dimensioned for a very large number of variables by merely changing a few cards.

Because the program calculates a matrix inverse, it must be noted that not all matrices can be accurately inverted. Such singular and "ill-conditioned" matrices are usually caused by the fact that one or more variables are linearly dependent on other variables in the matrix. Such linear dependencies can often be found by examining the inverse matrix for unusually large elements.

At present, the program is written in single precision, and it has performed well. However, users might consider converting the program to double precision by redeclaring the arrays double precision and by changing a few square-root functions from SQRT to DSQRT.

Availability. A program listing, a listing of sample data, and a user's manual may be obtained without charge by writing to Bernard S. Gorman or Louis H. Primavera, Department of Psychology, Hofstra University, Hempstead, New York 11550.

\section{REFERENCES}

Darlington, R. B. Multiple regression in psychological research and practice. Psychological Bulletin, 1968, 69, 161-182.

Dubois, P. H., \& Manning, W. H. An analytically meaningful approach to matrix inversion. Educational and Psychological Measurement, 1960, 20, 705-713.

Fox, K. A. Intermediate economic statistics. New York: Wiley, 1968.

Overall, J. E., \& KLetт, C. J. Applied multivariate analysis. New York: McGraw-Hill, 1972.

(Accepted for publication May 7, 1980.) 